\section{PERIPHERAL VASCULAR AUTOREGULATION DURING DUCTAL CLOSURE}

\author{
C. Hammerman ${ }^{1}$, A. Bin-Nun ${ }^{2}$
}

\begin{abstract}
${ }^{1}$ Neonatology, Hebrew University Faculty of Medicine, ${ }^{2}$ Neonatology, Shaare Zedek Med Ctr, Jerusalem, Israel
\end{abstract}

Background/aims: Tissue blood flow reflects the balance between perfusion pressure and local vascular resistance. The body must balance local needs against needs of central organs considered more critical. When perfusion is disrupted (eg PDA) fine control must be accomplished by variations in relative vascular resistance (RVR).

We looked at serial RVR changes in the anterior cerebral artery, superior mesenteric artery and right renal artery in infants being treated for PDA.

Design/methods: RVR was calculated as mean systemic blood pressure divided by mean local blood flow velocity. Flow velocities were studied by Doppler ultrasonography in 57 preterm neonates $<1750 \mathrm{gm}$. before, during and after treatment for ductal closure. All of the infants were NPO during treatment.

\section{Results:}

\section{RVR [Baseline, $24 \mathrm{hr}, 48 \mathrm{hr}]$}

Cerebral $2.7 \pm 1.1,3.6 \pm 1.4^{*}, 3.9 \pm 1.5^{*}$

Renal 2.2 [1.3,3.7], $2.8[2.3,4.1]^{*}, 2.7[1.7,4.1]$

Mesenteric $2.8[1.8,4.3]$, $3.0[2.0,4.1], 3.1[1.8,4.5]^{*}$

$p<0.05$ as compared with baseline

Conclusions: RVR reflects the balance of systemic vs.local flow, the essence of autoregulation. Dcreased RVR could represent a physiologic attempt to improve organ perfusion during hypotension, while increased RVR might reflect an attempt to protect susceptible organs against hypertension. To preserve blood flow, RVR would be expected to decrease in vasculature compromised by ductal shunting. Conversely, with ductal closure, increased RVR would be expected. We observed this expected response most significantly in the cerebral vasculature, implying, that this vasculature most adept at autoregulation. The mesenteric vasculature showed no autoregulatory response, which may shed further light on the association between NEC and PDA.

\section{THE EFFECT OF HUMAN RED CELLS STORAGE TIME ON THE MICROCIRCULATION OF PRETERM INFANTS}

Z. Mormanova ${ }^{1,2}$, K. Schottmeyer ${ }^{1}$, S. Hiedl ${ }^{1}$, O. Genzel - Boroviczény

${ }^{1}$ Neonatology, Department of Pediatrics, LMU, Munich, Germany, ${ }^{2}$ Department of

Pathophysiology, 2. medical faculty, Charles University, Prague, Czech Republic

Background: Red blood cell (RBC) transfusion practices for neonates are controversial, variable, and based on limited scientific information. To decrease donor exposure satellite packs are used leading to the transfusion of older RBCs. Transfusion of stored RBCs in a hemodiluted animal resulted in significant malperfused and underoxygenated microvasculature that was not detected at the systemic level.

Objective: Does the age of transfused erythrocytes influence functional vessel density (FVD) in the skin in premature infants?

Design/methods: 17 preterm infants with birth weight $<1500 \mathrm{~g}$ received $\mathrm{RBC}$ transfusion, 11 infants repeatedly from the same donor. The age of RBCs at repeated transfusions was significant higher than at the first transfusion.

Using Sidestream Dark Field Imaging all preterm infants were examined before and 2, 24 and 48 hours after transfusion. Images were blinded and analyzed with Microvision Analysis Software.

Results: FVD before first and repeated transfusion did not differ. In the $2^{\text {nd }}, 24^{\text {th }}$ and $48^{\text {th }}$ hour after the treatment the Functional Vessel Density (FVD) was significantly lower after the repeated transfusion with longer storage time ( $2^{\text {nd }}: 250$ vs. $238 \mathrm{~cm} / \mathrm{cm}^{2}$, $p=0.045 ; 24^{\text {th: }}: 259$ vs. $231 \mathrm{~cm}^{-\mathrm{cm}^{2}}, \mathrm{p}=0.001 ; 48^{\text {th }}$ : 234 vs. $220 \mathrm{~cm} / \mathrm{cm}^{2}, \mathrm{p}=0.035$ ).

The proportion of vessels with altered sluggish blood flow was also significantly higher when older RBCs were transfused (24h: $0.3 \%$ vs $2.5 \%, p=0.017 ; 48 \mathrm{~h}$ : $1 \%$ vs. $3.3 \%, p=0.013)$.

Conclusions: Similar to the animal model longer storage of the red blood cells reduces functional vessel density in the skin of premature infants and more vessels display sluggish blood flow. 